\title{
STUDY ON LA-RING BY THEIR INTUITIONISTIC FUZZY IDEALS
}

\section{NASREEN KAUSAR ${ }^{1 *}$, MESHARI ALESEMI ${ }^{2}$, SALAHUDDIN $^{2}$, MOHAMMAD MUNIR ${ }^{3}$}

\author{
${ }^{1}$ Department of Mathematics and Statistics, University of Agriculture, Faisalabad, Pakistán. \\ ${ }^{2}$ Department of Mathematics, Jazan University, Jazan, K. S. A. \\ ${ }^{3}$ Department of Mathematics, Government Postgraduate College, Abbottabad, Pakistán. \\ *Corresponding author. E-mail: kausar.nasreen57@gmail.com
}

DOI: $10.20948 /$ mathmontis-2020-47-3

Summary. In this paper, we extend the characterizations of Kuroki [17], by initiating the concept of intuitionistic fuzzy left ( resp. right, interior, quasi-, bi-, generalized bi-) ideals in a class of non-associative and non-commutative rings (LA-ring). We characterize regular (intra-regular, both regular and intra-regular) LA-rings in terms such ideals.

\section{INTRODUCTION}

In ternary operations, the commutative law is given by $a b c=c b a$. Kazim and Naseerudin [7], have generalized this notion by introducing the parenthesis on the left side of this equation to get a new pseudo associative law, that is $(a b) c=(c b) a$. This law $(a b) c=(c b) a$ is called the left invertive law. A groupoid $S$ is called a left almost semigroup (abbreviated as LA-semi-group) if it satisfies the left invertive law. An LA-semi-group is a midway structure between a commutative semigroup and a groupoid.

A groupoid $S$ is said to be medial ( resp. paramedial) if $(a b)(c d)=(a c)(b d)$ ( resp. $(a b)(c d)=(d b)(c a))$. An LA-semi-group is medial, but in general an LA-semi-group needs not to be paramedial. Every LA-semi-group with left identity is paramedial and also satisfies $a(b c)=b(a c),(a b)(c d)=(d c)(b a)$.

Kamran [16], extended the notion of LA-semi-group to the left almost group (LAgroup). An LA-semi-group $G$ is called a left almost group, if there exists a left identity $e \in G$ such that $e a=a$ for all $a \in G$ and for every $a \in G$ there exists $b \in G$ such that $b a=e$.

Shah et al. [22], by a left almost ring, mean a non-empty set $R$ with at least two elements such that $(R,+)$ is an LA-group, $(R, \cdot)$ is an LA-semi-group, both left and right distributive laws hold. For example, from a commutative ring $(R,+, \cdot)$, we can always obtain an LA-ring $(R, \oplus, \cdot)$ by defining for all $a, b \in R, a \oplus b=b-a$ and $a \cdot b$ is same as in the ring. Although the structure is non-associative and non-commutative, nevertheless, it possesses many interesting properties which we usually find in associative and commutative algebraic structures.

A non-empty subset $A$ of $R$ is called an LA-subring of $R$ if $a-b \in A$ and $a b \in A$ for all

2010 Mathematics Subject Classification: 97H20, 97H40, 94D05.

Key words and Phrases: Intuitionistic fuzzy left ( right, interior, quasi-, bi-, generalized bi-) ideals, regular (intra-regular) LA-rings. 
$a, b \in A . \quad A$ is called a left (resp. right) ideal of $R$ if $(A,+)$ is an LA-group and $R A \subseteq A$ (resp. $A R \subseteq A$ ). $A$ is called an ideal of $R$ if it is both a left ideal and a right ideal of $R$.

A non-empty subset $A$ of $R$ is called an interior ideal of $R$ if $(A,+)$ is an LA-group and $(R A) R \subseteq A$. A non-empty subset $A$ of $R$ is called a quasi-ideal of $R$ if $(A,+)$ is an LAgroup and $A R \cap R A \subseteq A$. An LA-subring $A$ of $R$ is called a bi-ideal of $R$ if $(A R) A \subseteq A$. A non-empty subset $A$ of $R$ is called a generalized bi-ideal of $R$ if $(A,+)$ is an LA-group and $(A R) A \subseteq A$.

We will define the concept of intuitionistic fuzzy left ( resp. right, interior, quasi-, bi-, generalized bi- ) ideals of an LA-ring $R$. We will establish a study by discussing the different properties of such ideals. We will characterize regular ( resp. intra-regular, both regular and intra-regular) LA-rings by the properties of intuitionistic fuzzy (left, right, quasi-, bi-, generalized bi- ) ideals such ideals.

\section{INTUITIONISTIC FUZZY IDEALS IN LA-RINGS}

After, the introduction of fuzzy set by Zadeh [24], several researchers explored on the generalization of the notion of fuzzy set. The concept of intuitionistic fuzzy set was introduced by Atanassov [1], as a generalization of the notion of fuzzy set. Liu [18], introduced the concept of fuzzy subrings and fuzzy ideals of a ring. Many authors have explored the theory of fuzzy rings (for example [3, 9, 11-15, 18, 19-20, 23]). Gupta and Kantroo [4], gave the idea of intrinsic product of fuzzy subsets of a ring. Kuroki [17], characterized regular (intra-regular, both regular and intra-regular) rings in terms of fuzzy left ( right, quasi, bi- ) ideals.

An intuitionistic fuzzy set (briefly, IFS ) $A$ in a non-empty set $X$ is an object having the form $A=\left\{\left(x, \mu_{A}(x), \gamma_{A}(x)\right): x \in X\right\}$, where the functions $\mu_{A}: X \rightarrow[0,1]$ and $\gamma_{A}: X \rightarrow[0,1]$ denote the degree of membership and the degree of non-membership, respectively and $0 \leq \mu_{A}(x)+\gamma_{A}(x) \leq 1$ for all $x \in X[1]$.

An intuitionistic fuzzy set $A=\left\{\left(x, \mu_{A}(x), \gamma_{A}(x)\right): x \in X\right\}$ in $X$ can be identified to be an ordered pair $\left(\mu_{A}, \gamma_{A}\right)$ in $I^{X} \times I^{X}$, where $I^{X}$ is the set of all functions from $X$ to $[0,1]$.

For the sake of simplicity, we shall use the symbol $A=\left(\mu_{A}, \gamma_{A}\right)$ for the IFS $A=\left\{\left(x, \mu_{A}(x), \gamma_{A}(x)\right): \quad x \in X\right\}$.

Banerjee and Basnet [2] and Hur et al. [6], initiated the notion of intuitionistic fuzzy subrings and intuitionistic fuzzy ideals of a ring. Subsequently many authors studied the intuitionistic fuzzy subrings and intuitionistic fuzzy ideals of a ring by describing the different properties ( see [5]). Shah et al. [21, 22] initiated the concept of intuitionistic fuzzy normal subrings over a non-associative ring and also characterized the non-associative rings by their intuitionistic fuzzy bi-ideals in [8]. Kausar [10] explored the notion of direct product of finite intuitionistic anti fuzzy normal subrings over non-associative rings. 
We initiate the notion of intuitionistic fuzzy left (resp. right, interior, quasi-, bi-, generalized bi- ) ideals of an LA-ring $R$.

An intuitionistic fuzzy set ( IFS ) $A=\left(\mu_{A}, \gamma_{A}\right)$ of an LA-ring $R$ is called an intuitionistic fuzzy LA-subring of $R$ if

(1) $\mu_{A}(x-y) \geq \min \left\{\mu_{A}(x), \mu_{A}(y)\right\}$,

(2) $\gamma_{A}(x-y) \leq \max \left\{\gamma_{A}(x), \gamma_{A}(y)\right\}$

(3) $\mu_{A}(x y) \geq \min \left\{\mu_{A}(x), \mu_{A}(y)\right\}$,

(4) $\gamma_{A}(x y) \leq \max \left\{\gamma_{A}(x), \gamma_{A}(y)\right\}$, for all $x, y \in R$.

An IFS $A=\left(\mu_{A}, \gamma_{A}\right)$ of an LA-ring $R$ is called an intuitionistic fuzzy left ideal of $R$ if

(1) $\mu_{A}(x-y) \geq \min \left\{\mu_{A}(x), \mu_{A}(y)\right\}$,

(2) $\gamma_{A}(x-y) \leq \max \left\{\gamma_{A}(x), \gamma_{A}(y)\right\}$

(3) $\mu_{A}(x y) \geq \mu_{A}(y)$

(4) $\gamma_{A}(x y) \leq \gamma_{A}(y)$, for all $x, y \in R$.

An IFS $A=\left(\mu_{A}, \gamma_{A}\right)$ of an LA-ring $R$ is called an intuitionistic fuzzy right ideal of $R$ if

(1) $\mu_{A}(x-y) \geq \min \left\{\mu_{A}(x), \mu_{A}(y)\right\}$,

(2) $\gamma_{A}(x-y) \leq \max \left\{\gamma_{A}(x), \gamma_{A}(y)\right\}$

(3) $\mu_{A}(x y) \geq \mu_{A}(x)$

(4) $\gamma_{A}(x y) \leq \gamma_{A}(x)$, for all $x, y \in R$.

An IFS $A=\left(\mu_{A}, \gamma_{A}\right)$ of $R$ is called an intuitionistic fuzzy ideal of an LA-ring $R$ if it is both an intuitionistic fuzzy left ideal and an intuitionistic fuzzy right ideal of $R$.

Let $A$ be a non-empty subset of an LA-ring $R$. Then the intuitionistic characteristic of $A$ is denoted by $\chi_{A}=\left\langle\mu_{\chi_{A}}, \gamma_{\chi_{A}}\right\rangle$ and defined by

$$
\mu_{\chi_{A}}(x)=\left\{\begin{array}{l}
1 \text { if } x \in A \\
0 \text { if } x \notin A
\end{array} \text { and } \gamma_{\chi_{A}}(x)=\left\{\begin{array}{l}
0 \text { if } x \in A \\
1 \text { if } x \notin A
\end{array}\right.\right.
$$

We note that an LA-ring $R$ can be considered an intuitionistic fuzzy set of itself and we write $R=I_{R}$, i.e., $R(x)=\left(\mu_{R}, \gamma_{R}\right)=(1,0)$ for all $x \in R$.

Let $A$ and $B$ be two intuitionistic fuzzy sets of an LA-ring $R$. Then

(1) $A \subseteq B \Leftrightarrow \mu_{A} \subseteq \mu_{B}$ and $\gamma_{A} \supseteq \gamma_{B}$,

(2) $A=B \Leftrightarrow A \subseteq B$ and $B \subseteq A$,

(3) $A^{c}=\left(\gamma_{A}, \mu_{A}\right)$, 
(4) $A \cap B=\left(\mu_{A} \wedge \mu_{B}, \gamma_{A} \vee \gamma_{B}\right)=\left(\mu_{A \wedge B}, \gamma_{A \vee B}\right)$,

(5) $A \cup B=\left(\mu_{A} \vee \mu_{B}, \gamma_{A} \wedge \gamma_{B}\right)=\left(\mu_{A \vee B}, \gamma_{A \wedge B}\right)$,

(6) $0 \approx=(0,1), 1 \approx=(1,0)$.

The product of $A=\left(\mu_{A}, \gamma_{A}\right)$ and $B=\left(\mu_{B}, \gamma_{B}\right)$ is denoted by $A \circ B=\left(\mu_{A} \circ \mu_{B}, \gamma_{A} \circ \gamma_{B}\right)$ and defined as:

$$
\begin{aligned}
\left(\mu_{A} \circ \mu_{B}\right)(x) & =\left\{\begin{array}{cc}
\vee \sum_{x=\sum_{i=1}^{n} a_{i} b_{i}}\left\{\wedge_{i=1}^{n}\left\{\mu_{A}\left(a_{i}\right) \wedge \mu_{B}\left(b_{i}\right)\right\}\right\} & \text { if } x=\sum_{i=1}^{n} a_{i} b_{i}, a_{i}, b_{i} \in R \\
0 & \text { if } x \neq \sum_{i=1}^{n} a_{i} b_{i}
\end{array}\right. \\
\text { and }\left(\gamma_{A} \circ \gamma_{B}\right)(x) & =\left\{\begin{array}{cc}
\wedge \sum_{x=\sum_{i=1}^{n} a_{i} b_{i}}\left\{\vee_{i=1}^{n}\left\{\gamma_{A}\left(a_{i}\right) \vee \gamma_{B}\left(b_{i}\right)\right\}\right\} & \text { if } x=\sum_{i=1}^{n} a_{i} b_{i}, a_{i}, b_{i} \in R \\
1 & \text { if } x \neq \sum_{i=1}^{n} a_{i} b_{i}
\end{array}\right.
\end{aligned}
$$

An IFS $A=\left(\mu_{A}, \gamma_{A}\right)$ of an LA-ring $R$ is called an intuitionistic fuzzy interior ideal of $R$ if

(1) $\mu_{A}(x-y) \geq \mu_{A}(x) \wedge \mu_{A}(y)$,

(2) $\gamma_{A}(x-y) \leq \gamma_{A}(x) \vee \gamma_{A}(y)$,

(3) $\mu_{A}((x y) z) \geq \mu_{A}(y)$,

(4) $\gamma_{A}((x y) z) \leq \gamma_{A}(y)$, for all $x, y, z \in R$.

An IFS $A=\left(\mu_{A}, \gamma_{A}\right)$ of an LA-ring $R$ is called an intuitionistic fuzzy quasi-ideal of $R$ if

(1) $\left(\mu_{A} \circ R\right) \cap\left(R \circ \mu_{A}\right) \subseteq \mu_{A}$,

(2) $\left(\gamma_{A} \circ R\right) \cup\left(R \circ \gamma_{A}\right) \supseteq \gamma_{A}$,

(3) $\mu_{A}(x-y) \geq \mu_{A}(x) \wedge \mu_{A}(y)$,

(4) $\gamma_{A}(x-y) \leq \gamma_{A}(x) \vee \gamma_{A}(y)$, for all $x, y \in R$.

An Intuitionistic fuzzy LA-subring $A=\left(\mu_{A}, \gamma_{A}\right)$ of an LA-ring $R$ is called an intuitionistic fuzzy bi-ideal of $R$ if

(1) $\mu_{A}((x y) z) \geq \mu_{A}(x) \wedge \mu_{A}(z)$,

(2) $\gamma_{A}((x y) z) \leq \gamma_{A}(x) \vee \gamma_{A}(z)$, for all $x, y, z \in R$.

An IFS $A=\left(\mu_{A}, \gamma_{A}\right)$ of an LA-ring $R$ is called an intuitionistic fuzzy generalized bi-ideal of $R$ if

(1) $\mu_{A}(x-y) \geq \mu_{A}(x) \wedge \mu_{A}(y)$, 
(2) $\gamma_{A}(x-y) \leq \gamma_{A}(x) \vee \gamma_{A}(y)$,

(3) $\mu_{A}((x y) z) \geq \mu_{A}(x) \wedge \mu_{A}(z)$,

(4) $\gamma_{A}((x y) z) \leq \gamma_{A}(x) \vee \gamma_{A}(z)$, for all $x, y, z \in R$.

An intuitionistic fuzzy ideal $A=\left(\mu_{A}, \gamma_{A}\right)$ of an LA-ring $R$ is called an intuitionistic fuzzy idempotent if $\mu_{A} \circ \mu_{A}=\mu_{A}$ and $\gamma_{A} \circ \gamma_{A}=\gamma_{A}$.

Now we give some imperative properties of such ideals of an LA-ring $R$, which will be very helpful in later sections.

Lemma 2.1: Let $R$ be an LA-ring. Then the following properties hold:

(1) $(A \circ B) \circ C=(C \circ B) \circ A$,

(2) $(A \circ B) \circ(C \circ D)=(A \circ C) \circ(B \circ D)$,

(3) $A \circ(B \circ C)=B \circ(A \circ C)$,

(4) $(A \circ B) \circ(C \circ D)=(D \circ B) \circ(C \circ A)$,

(5) $(A \circ B) \circ(C \circ D)=(D \circ C) \circ(B \circ A)$, for all intuitionistic fuzzy sets $A, B, C$ and $D$ of $R$.

Proof: Obvious.

Theorem 2.2: Let $A$ and $B$ be two non-empty subsets of an LA-ring $R$. then the following properties hold:

(1) If $A \subseteq B$ then $\chi_{A} \subseteq \chi_{B}$.

(2) $\chi_{A} \circ \chi_{B}=\chi_{A B}$.

(4) $\chi_{A} \cap \chi_{B}=\chi_{A \cap B}$.

Proof: (1) Suppose that $A \subseteq B$ and $a \in R$. If $a \in A$, this implies that $a \in B$. Thus $\mu_{\chi_{A}}(a)=1=\mu_{\chi_{B}}(a)$ and $\gamma_{\chi_{A}}(a)=0=\gamma_{\chi_{B}}(a)$, i.e., $\chi_{A} \subseteq \chi_{B}$.

If $a \notin A$, and $a \notin B$. Thus $\mu_{\chi_{A}}(a)=0=\mu_{\chi_{B}}(a)$ and $\gamma_{\chi_{A}}(a)=1=\gamma_{\chi_{B}}(a)$, i.e., $\chi_{A} \subseteq \chi_{B}$.

If $\alpha \notin A$ and $\alpha \in B$. Thus $\mu_{\chi A}(\alpha)=0$ and $\mu_{\chi B}(\alpha)=1$ and $\gamma_{\chi A}(\alpha)=1$ and $\gamma_{\chi B}(\alpha)=0$, i.e.,

(2) Let $x \in R$ and $x \in A B$. This means that $x=a b$ for some $\alpha \in A$ and $b \in B$.

Now

$$
\begin{aligned}
\left(\mu_{\chi_{A}} \circ \mu_{\chi_{B}}\right)(x) & =\vee_{x=\sum_{i=1}^{n} a_{i} b_{i}}\left\{\wedge_{i=1}^{n}\left\{\mu_{\chi_{A}}\left(a_{i}\right) \wedge \mu_{\chi_{B}}\left(b_{i}\right)\right\}\right\} \\
& \geq \mu_{\chi_{A}}(a) \wedge \mu_{\chi_{B}}(b)=1 \wedge 1=1=\mu_{\chi_{A B}}(x) \\
\text { and }\left(\gamma_{\chi_{A}} \circ \gamma_{\chi_{B}}\right)(x) & =\wedge_{x=\sum_{i=1}^{n} a_{i} b_{i}}\left\{\vee_{i=1}^{n}\left\{\gamma_{\chi_{A}}\left(a_{i}\right) \vee \gamma_{\chi_{B}}\left(b_{i}\right)\right\}\right\} \\
& \leq \gamma_{\chi_{A}}(a) \vee \gamma_{\chi_{B}}(b)=0 \vee 0=0=\gamma_{\chi_{A B}}(x) .
\end{aligned}
$$

If $x \notin A B$, i.e., $x \neq a b$ for all $a \in A$ and $b \in B$. Then there are two cases. 
(i) If $x=u v$ for some $u, v \in R$, then

$$
\begin{aligned}
\left(\mu_{\chi_{A}} \circ \mu_{\chi_{B}}\right)(x) & =\vee_{x=\sum_{i=1}^{n} a_{i} b_{i}}\left\{\wedge_{i=1}^{n}\left\{\mu_{\chi_{A}}\left(a_{i}\right) \wedge \mu_{\chi_{B}}\left(b_{i}\right)\right\}\right\} \\
& =0 \wedge 0=0=\mu_{\chi_{A B}}(x) \\
\text { and }\left(\gamma_{\chi_{A}} \circ \gamma_{\chi_{B}}\right)(x) & =\wedge_{x=\sum_{i=1}^{n} a_{i} b_{i}}\left\{\vee_{i=1}^{n}\left\{\gamma_{\chi_{A}}\left(a_{i}\right) \vee \gamma_{\chi_{B}}\left(b_{i}\right)\right\}\right\} \\
& =1 \vee 1=1=\gamma_{\chi_{A B}}(x) .
\end{aligned}
$$

(ii) If $x \neq u v$ for all $u, v \in R$, then obviously $\left(\chi_{A} \circ \chi_{B}\right)(x)=0=\chi_{A B}(x)$. Hence $\chi_{A} \circ \chi_{B}=\chi_{A B}$.

Similarly, we can prove (3) and (4).

Theorem 2.3: Let $A$ be a non-empty subset of an LA-ring $R$. then the following properties hold.

(1) $A$ is an LA-subring of $R$ if and only if $\chi_{A}$ is an intuitionistic fuzzy LA-subring of $R$.

(2) $A$ is a left (resp. right, two-sided) ideal of $R$ if and only if $\chi_{A}$ is an intuitionistic fuzzy left ( resp. right, two-sided) ideal of $R$.

Proof: (1) Let $A$ be an LA-subring of $R$ and $a, b \in R$. If $a, b \in A$, then by definition $\mu_{A}(a)=1=\mu_{A}(b)$ and $\gamma_{A}(a)=0=\gamma_{A}(b)$. Since $a-b$ and $a b \in A, A$ being an LAsubring of $R$, this implies that $\mu_{A}(a-b)=1=\mu_{A}(a b)$ and $\gamma_{A}(a-b)=0=\gamma_{A}(a b)$. Thus

$$
\begin{aligned}
\mu_{A}(a-b) & \geq \mu_{A}(a) \wedge \mu_{A}(b), \quad \mu_{A}(a b) \geq \mu_{A}(a) \wedge \mu_{A}(b) \\
\text { and } \gamma_{A}(a-b) & \leq \gamma_{A}(a) \vee \gamma_{A}(b), \gamma_{A}(a b) \leq \gamma_{A}(a) \vee \gamma_{A}(b) .
\end{aligned}
$$

Similarly, we have

$$
\begin{aligned}
\mu_{A}(a-b) & \geq \mu_{A}(a) \wedge \mu_{A}(b), \quad \mu_{A}(a b) \geq \mu_{A}(a) \wedge \mu_{A}(b) . \\
\text { and } \gamma_{A}(a-b) & \leq \gamma_{A}(a) \vee \gamma_{A}(b), \gamma_{A}(a b) \leq \gamma_{A}(a) \vee \gamma_{A}(b) .
\end{aligned}
$$

when $a, b \notin A$. Hence $\chi_{A}$ is an intuitionistic fuzzy LA-subring of $R$.

Conversely, suppose that $\chi_{A}$ is an intuitionistic fuzzy LA-subring of $R$ and let $a, b \in A$. This means that $\mu_{A}(a)=1=\mu_{A}(b)$ and $\gamma_{A}(a)=0=\gamma_{A}(b)$. Since

$$
\begin{aligned}
& \mu_{A}(a-b) \geq \mu_{A}(a) \wedge \mu_{A}(b)=1 \wedge 1=1, \\
& \mu_{A}(a b) \geq \mu_{A}(a) \wedge \mu_{A}(b)=1 \wedge 1=1, \\
& \gamma_{A}(a-b) \leq \gamma_{A}(a) \vee \gamma_{A}(b)=0 \vee 0=0, \\
& \gamma_{A}(a b) \leq \gamma_{A}(a) \vee \gamma_{A}(b)=0 \vee 0=0,
\end{aligned}
$$

$\chi_{A}$ being an intuitionistic fuzzy LA-subring of $R$. Thus $\mu_{A}(a-b)=1=\mu_{A}(a b)$ and $\gamma_{A}(a-b)=0=\gamma_{A}(a b)$, i.e., $a-b$ and $a b \in A$. Hence $A$ is an LA-subring of $R$. 
(2) Let $A$ be a left ideal of $R$ and $a, b \in R$. If $a, b \in A$, then by definition $\mu_{A}(a)=1=\mu_{A}(b)$ and $\gamma_{A}(a)=0=\gamma_{A}(b)$. Since $a-b$ and $a b \in A, A$ being a left ideal of $R$, this implies that $\mu_{A}(a-b)=1=\mu_{A}(a b)$ and $\gamma_{A}(a-b)=0=\gamma_{A}(a b)$. Thus

$$
\begin{aligned}
\mu_{A}(a-b) & \geq \mu_{A}(a) \wedge \mu_{A}(b), \mu_{A}(a b) \geq \mu_{A}(b) \\
\text { and } \gamma_{A}(a-b) & \leq \gamma_{A}(a) \vee \gamma_{A}(b), \gamma_{A}(a b) \leq \gamma_{A}(b) .
\end{aligned}
$$

Similarly, we have

$$
\begin{aligned}
\mu_{A}(a-b) & \geq \mu_{A}(a) \wedge \mu_{A}(b), \mu_{A}(a b) \geq \mu_{A}(b) \\
\text { and } \gamma_{A}(a-b) & \leq \gamma_{A}(a) \vee \gamma_{A}(b), \gamma_{A}(a b) \leq \gamma_{A}(b) .
\end{aligned}
$$

when $a, b \notin A$. Therefore $\chi_{A}$ is an intuitionistic fuzzy left ideal of $R$.

Conversely, assume that $\chi_{A}$ is an intuitionistic fuzzy left ideal of $R$ and let

$a, b \in A$ and $z \in R$. This means that $\mu_{A}(a)=1=\mu_{A}(b)$ and $\gamma_{A}(a)=0=\gamma_{A}(b)$. Since

$$
\begin{aligned}
\mu_{A}(a-b) & \geq \mu_{A}(a) \wedge \mu_{A}(b)=1 \wedge 1=1, \\
\mu_{A}(z b) & \geq \mu_{A}(b)=1, \\
\gamma_{A}(a-b) & \leq \gamma_{A}(a) \vee \gamma_{A}(b)=0 \vee 0=0, \\
\gamma_{A}(z b) & \leq \gamma_{A}(b)=0,
\end{aligned}
$$

$\chi_{A}$ being an intuitionistic fuzzy left ideal of $R$. Thus $\mu_{A}(a-b)=1=\mu_{A}(z b)$ and $\gamma_{A}(a-b)=0=\gamma_{A}(z b)$, i.e., $a-b$ and $z b \in A$. Therefore $A$ is a left ideal of $R$.

Remark 2.4: $(i) A$ is an additive LA-subgroup of $R$ if and only if $\chi_{A}$ is an intuitionistic fuzzy additive LA-subgroup of $R$.

(ii) $A$ is an LA-subsemigroup of $R$ if and only if $\chi_{A}$ is an intuitionistic fuzzy LAsubsemigroup of $R$.

Lemma 2.5: If $A$ and $B$ are two intuitionistic fuzzy LA-subrings ( resp. (left, right, twosided ) ideals ) of an LA-ring $R$, then $A \cap B$ is also an intuitionistic fuzzy LA-subring ( resp. ( left, right, two-sided) ideal) of $R$.

\section{Proof: Obvious.}

Lemma 2.6: If $A$ and $B$ are two intuitionistic fuzzy LA-subrings of an LA-ring $R$, then $A \circ B$ is also an intuitionistic fuzzy LA-subring of $R$.

Proof: Let $A=\left(\mu_{A}, \gamma_{A}\right)$ and $B=\left(\mu_{B}, \gamma_{B}\right)$ be two intuitionistic fuzzy LA-subrings of $R$. We have to show that $A \circ B$ is also an intuitionistic fuzzy LA-subring of $R$. Now

$$
\begin{aligned}
\left(\mu_{A} \circ \mu_{B}\right)^{2} & =\left(\mu_{A} \circ \mu_{B}\right) \circ\left(\mu_{A} \circ \mu_{B}\right)=\left(\mu_{A} \circ \mu_{A}\right) \circ\left(\mu_{B} \circ \mu_{B}\right) \subseteq \mu_{A} \circ \mu_{B} \\
\text { and }\left(\gamma_{A} \circ \gamma_{B}\right)^{2} & =\left(\gamma_{A} \circ \gamma_{B}\right) \circ\left(\gamma_{A} \circ \gamma_{B}\right)=\left(\gamma_{A} \circ \gamma_{A}\right) \circ\left(\gamma_{B} \circ \gamma_{B}\right) \supseteq \gamma_{A} \circ \gamma_{B} .
\end{aligned}
$$

Since $\mu_{B}-\mu_{B} \subseteq \mu_{B}$ and $\gamma_{B}-\gamma_{B} \supseteq \gamma_{B}, B=\left(\mu_{B}, \gamma_{B}\right)$ being an intuitionistic fuzzy LA-subring of $R$. This implies that $\mu_{A} \circ\left(\mu_{B}-\mu_{B}\right) \subseteq \mu_{A} \circ \mu_{B}$ and 
$\gamma_{A} \circ\left(\gamma_{B}-\gamma_{B}\right) \supseteq \gamma_{A} \circ \gamma_{B}$, i.e., $\quad \mu_{A} \circ \mu_{B}-\mu_{A} \circ \mu_{B} \subseteq \mu_{A} \circ \mu_{B} \quad$ and $\gamma_{A} \circ \gamma_{B}-\gamma_{A} \circ \gamma_{B} \supseteq \gamma_{A} \circ \gamma_{B}$. Therefore $A \circ B$ is an intuitionistic fuzzy LA-subring of $R$. Remark 2.7: If $A$ is an intuitionistic fuzzy LA-subring of an LA-ring $R$, then $A \circ A$ is also an intuitionistic fuzzy LA-subring of $R$.

Lemma 2.8: Let $R$ be an LA-ring with left identity $e$. Then $R R=R$ and $e R=R=\operatorname{Re}$. Proof: Since $R R \subseteq R$ and $x=e x \in R R$, where $x \in R$, i.e., $R R=R$. Since $e$ is the left identity of $R$, i.e., $e R=R$. Now $\operatorname{Re}=(R R) e=(e R) R=R R=R$.

Lemma 2.9: Let $R$ be an LA-ring with left identity $e$. Then every intuitionistic fuzzy right ideal of $R$ is an intuitionistic fuzzy ideal of $R$.

Proof: Let $A=\left(\mu_{A}, \gamma_{A}\right)$ be an intuitionistic fuzzy right ideal of $R$ and $x, y \in R$. Now

$$
\begin{aligned}
\mu_{A}(x y) & =\mu_{A}((e x) y)=\mu_{A}((y x) e) \geq \mu_{A}(y x) \geq \mu_{A}(y) \\
\text { and } \gamma_{A}(x y) & =\gamma_{A}((e x) y)=\gamma_{A}((y x) e) \leq \gamma_{A}(y x) \leq \gamma_{A}(y) .
\end{aligned}
$$

Thus $A$ is an intuitionistic fuzzy ideal of $R$.

Lemma 2.10: If $A$ and $B$ are two intuitionistic fuzzy left ( resp. right) ideals of an LA$\operatorname{ring} R$ with left identity $e$, then $A \circ B$ is also an intuitionistic fuzzy left (resp. right) ideal of $R$.

Proof: Let $A=\left(\mu_{A}, \gamma_{A}\right)$ and $B=\left(\mu_{B}, \gamma_{B}\right)$ be two intuitionistic fuzzy left ideals of $R$. We have to show that $A \circ B$ is also an intuitionistic fuzzy left ideal of $R$. Since $\mu_{A} \circ \mu_{B}-\mu_{A} \circ \mu_{B} \subseteq \mu_{A} \circ \mu_{B}$ and $\gamma_{A} \circ \gamma_{B}-\gamma_{A} \circ \gamma_{B} \supseteq \gamma_{A} \circ \gamma_{B}$. Now

$$
\begin{aligned}
R \circ\left(\mu_{A} \circ \mu_{B}\right) & =(R \circ R) \circ\left(\mu_{A} \circ \mu_{B}\right)=\left(R \circ \mu_{A}\right) \circ\left(R \circ \mu_{B}\right) \subseteq\left(\mu_{A} \circ \mu_{B}\right) \\
\text { and } R \circ\left(\gamma_{A} \circ \gamma_{B}\right) & =(R \circ R) \circ\left(\gamma_{A} \circ \gamma_{B}\right)=\left(R \circ \gamma_{A}\right) \circ\left(R \circ \gamma_{A}\right) \supseteq\left(\gamma_{A} \circ \gamma_{B}\right) .
\end{aligned}
$$

Hence $A \circ B$ is an intuitionistic fuzzy left ideal of $R$.

Remark 2.11: If $A$ is an intuitionistic fuzzy left (resp. right) ideal of an LA-ring $R$ with left identity $e$, then $A \circ A$ is an intuitionistic fuzzy ideal of $R$.

Lemma 2.12: If $A$ and $B$ are two intuitionistic fuzzy ideals of an LA-ring $R$, then $A \circ B \subseteq A \cap B$.

Proof: Let $A=\left(\mu_{A}, \gamma_{A}\right)$ and $B=\left(\mu_{B}, \gamma_{B}\right)$ be two intuitionistic fuzzy ideals of $R$ and $x \in R$. If $x$ cannot expressible as $x=\sum_{i=1}^{n} a_{i} b_{i}$, where $a_{i}, b_{i} \in R$ and $n$ is any positive integer, then obviously $A \circ B \subseteq A \cap B$, otherwise we have

$$
\begin{aligned}
\left(\mu_{A} \circ \mu_{B}\right)(x) & =\vee_{x=\sum_{i=1}^{n} a_{i} b_{i}}\left\{\wedge_{i=1}^{n}\left\{\mu_{A}\left(a_{i}\right) \wedge \mu_{B}\left(b_{i}\right)\right\}\right\} \\
& \leq \vee_{x=\sum_{i=1}^{n} a_{i} b_{i}}\left\{\wedge_{i=1}^{n}\left\{\mu_{A}\left(a_{i} b_{i}\right) \wedge \mu_{B}\left(a_{i} b_{i}\right)\right\}\right\} \\
& =\vee_{x=\sum_{i=1}^{n} a_{i} b_{i}}\left\{\wedge_{i=1}^{n}\left(\mu_{A} \cap \mu_{B}\right)\left(a_{i} b_{i}\right)\right\}=\left(\mu_{A} \cap \mu_{B}\right)(x) . \\
& \Rightarrow \mu_{A} \circ \mu_{B} \subseteq \mu_{A} \cap \mu_{B} .
\end{aligned}
$$

Similarly, we can prove $\gamma_{A} \circ \gamma_{B} \supseteq \gamma_{A} \cup \gamma_{B}$. 
Therefore $A \circ B \subseteq A \cap B$ for all intuitionistic fuzzy ideals $A$ and $B$ of $R$.

Remark 2.13: If $A$ is an intuitionistic fuzzy ideal of an LA-ring $R$, then $A \circ A \subseteq A$.

Lemma 2.14: Let $R$ be an LA-ring. Then $A \circ B \subseteq A \cap B$ for every intuitionistic fuzzy right ideal $A$ and every intuitionistic fuzzy left ideal $B$ of $R$.

Proof: Same as Lemma 2.12

Theorem 2.15: Let $A$ be a non-empty subset of an LA-ring $R$. Then $A$ is an interior (resp. quasi-, bi-, generalized bi-) ideal of $R$ if and only if $\chi_{A}$ is an intuitionistic fuzzy interior (resp. quasi-, bi-, generalized bi-) ideal of $R$.

Proof: Let $A$ be an interior ideal of $R$, this implies that $A$ is an additive LA-subgroup of $R$. Then $\chi_{A}$ is an intuitionistic fuzzy additive LA-subgroup of $R$ by the Remark 2.4. Let $x, y, a \in R$. If $a \in A$, then by definition $\mu_{\chi_{A}}(a)=1$ and $\gamma_{\chi_{A}}(a)=0$. Since (xa) $y \in A, A$ being an interior ideal of $R$, this means that $\mu_{\chi_{A}}((x a) y)=1$ and $\gamma_{\chi_{A}}((x a) y)=0$. Thus $\mu_{\chi_{A}}((x a) y) \geq \mu_{\chi_{A}}(a)$ and $\gamma_{\chi_{A}}((x a) y) \leq \gamma_{\chi_{A}}(a)$. Similarly, we have $\mu_{\chi_{A}}((x a) y) \geq \mu_{\chi_{A}}(a)$ and $\gamma_{\chi_{A}}((x a) y) \leq \gamma_{\chi_{A}}(a)$, when $a \notin A$. Hence $\chi_{A}$ is an intuitionistic fuzzy interior ideal of $R$.

Conversely, suppose that $\chi_{A}$ is an intuitionistic fuzzy interior ideal of $R$, this means that $\chi_{A}$ is an intuitionistic fuzzy additive LA-subgroup of $R$. Then $A$ is an additive LA-subgroup of $R$ by the Remark 2.4. Let $x, y \in R$ and $a \in A$, so $\mu_{\chi_{A}}(a)=1$ and $\gamma_{\chi_{A}}(a)=0$. Since $\mu_{\chi_{A}}((x a) y) \geq \mu_{\chi_{A}}(a)=1$ and $\gamma_{\chi_{A}}((x a) y) \leq \gamma_{\chi_{A}}(a)=0, \quad \chi_{A}$ being an intuitionistic fuzzy interior ideal of $R$. Thus $\mu_{\chi_{A}}((x a) y)=1$ and $\gamma_{\chi_{A}}((x a) y)=0$, i.e., $\quad(x a) y \in A$. Hence $A$ is an interior ideal of $R$. Similarly, we can prove for (quasi-, bi-, generalized bi-) ideal.

Lemma 2.16: If $A$ and $B$ are two intuitionistic fuzzy bi-( resp. generalized bi-, quasi-, interior) ideals of an LA-ring $R$, then $A \cap B$ is also an intuitionistic fuzzy bi- (resp. generalized bi-, quasi-, interior) ideal of $R$.

Proof: Obvious.

Lemma 2.17: If $A$ and $B$ are two intuitionistic fuzzy bi- (resp. generalized bi-, interior) ideals of an LA-ring $R$ with left identity $e$, then $A \circ B$ is also an intuitionistic fuzzy bi( resp. generalized bi-, interior) ideal of $R$.

Proof: Let $A=\left(\mu_{A}, \gamma_{A}\right)$ and $B=\left(\mu_{B}, \gamma_{B}\right)$ be two intuitionistic fuzzy bi-ideals of $R$. We have to show that $A \circ B$ is also an intuitionistic fuzzy bi-ideal of $R$. Since $A$ and $B$ are two intuitionistic fuzzy LA-subrings of $R$, then $A \circ B$ is also an intuitionistic fuzzy LAsubring of $R$ by the Lemma 2.6. Now

$$
\begin{aligned}
\left(\left(\mu_{A} \circ \mu_{B}\right) \circ R\right) \circ\left(\mu_{A} \circ \mu_{B}\right) & =\left(\left(\mu_{A} \circ \mu_{B}\right) \circ(R \circ R)\right) \circ\left(\mu_{A} \circ \mu_{B}\right) \\
& =\left(\left(\mu_{A} \circ R\right) \circ\left(\mu_{B} \circ R\right)\right) \circ\left(\mu_{A} \circ \mu_{B}\right) \\
& =\left(\left(\mu_{A} \circ R\right) \circ \mu_{A}\right) \circ\left(\left(\mu_{B} \circ R\right) \circ \mu_{B}\right) \\
& \subseteq \mu_{A} \circ \mu_{B} .
\end{aligned}
$$


Similarly, we have $\left(\left(\gamma_{A} \circ \gamma_{B}\right) \circ R\right) \circ\left(\gamma_{A} \circ \gamma_{B}\right) \supseteq \gamma_{A} \circ \gamma_{B}$. Therefore $A \circ B$ is an intuitionistic fuzzy bi-ideal of $R$.

Lemma 2.18: Every intuitionistic fuzzy ideal of an LA-ring $R$ is an intuitionistic fuzzy interior ideal of $R$. The converse is not true in general.

Proof: Let $A=\left(\mu_{A}, \gamma_{A}\right)$ be an intuitionistic fuzzy ideal of $R$ and $x, y, z \in R$. Thus $\mu_{A}((x y) z)=\mu_{A}(x y) \geq \mu_{A}(y)$ and $\gamma_{A}((x y) z)=\gamma_{A}(x y) \leq \gamma_{A}(y)$. Hence $A$ is an intuitionistic fuzzy interior ideal of $R$.

The converse is not true in general, giving an example:

Example 2.19: Let $R=\{0,1,2,3,4,5,6,7\}$ is an LA-ring.

\begin{tabular}{|c|c|c|c|c|c|c|c|c|c|c|c|c|c|c|c|c|}
\hline t & $\begin{array}{ll}0 & 1\end{array}$ & 2 & 3 & 4 & 5 & 6 & 7 & & . & 0 & 1 & 2 & 3 & 4 & & 6 \\
\hline 0 & 1 & 2 & 3 & 4 & 5 & 6 & 7 & & 0 & 0 & 0 & 0 & 0 & 0 & ) & 0 \\
\hline 1 & 2 & 3 & 1 & 6 & 4 & 7 & 5 & & 1 & 0 & 4 & 4 & 0 & 0 & & 4 \\
\hline 2 & 1 & 0 & 2 & 5 & 7 & 4 & 6 & & 2 & 0 & 4 & 4 & 0 & 0 & 4 & 4 \\
\hline 3 & 2 & 1 & 0 & 7 & 6 & 5 & 4 & and & 3 & 0 & 0 & 0 & 0 & 0 & ) & 0 \\
\hline 4 & 5 & 6 & 7 & 0 & 1 & 2 & 3 & & 4 & 0 & 3 & 3 & 0 & 0 & 3 & 3 \\
\hline 5 & 6 & 7 & 5 & 2 & 0 & 3 & 1 & & 5 & 0 & 7 & 7 & 0 & 0 & 7 & 7 \\
\hline 6 & 5 & 4 & 6 & 1 & 3 & 0 & 2 & & 6 & 0 & 7 & 7 & 0 & 0 & 7 & 7 \\
\hline & 6 & 5 & 4 & 3 & 2 & 1 & 0 & & 7 & 0 & 3 & 3 & 0 & 0 & & 3 \\
\hline
\end{tabular}

Let $A=\left(\mu_{A}, \gamma_{A}\right)$ be an IFS of an LA-ring $R$. We define

$$
\mu_{A}(0)=\mu_{A}(4)=0.7, \quad \mu_{A}(1)=\mu_{A}(2)=\mu_{A}(3)=\mu_{A}(5)=\mu_{A}(6)=\mu_{A}(7)=0
$$

and $\gamma_{A}(0)=\gamma_{A}(4)=0, \quad \gamma_{A}(1)=\gamma_{A}(2)=\gamma_{A}(3)=\gamma_{A}(5)=\gamma_{A}(6)=\gamma_{A}(7)=0.7$.

$A=\left(\mu_{A}, \gamma_{A}\right)$ is an intuitionistic fuzzy interior ideal of $R$, but not an intuitionistic fuzzy ideal of $R$, because $A$ is not an intuitionistic fuzzy right ideal of $R$, as

$$
\begin{aligned}
\mu_{A}(41) & =\mu_{A}(3)=0 . \\
\mu_{A}(4) & =0.7 . \\
& \Rightarrow \mu_{A}(41) \geq \mu_{A}(4) . \\
\text { and } \gamma_{A}(41) & =\gamma_{A}(3)=0.7 . \\
\gamma_{A}(4) & =0 . \\
& \Rightarrow \gamma_{A}(41) \nsubseteq \gamma_{A}(4) .
\end{aligned}
$$

Proposition 2.20: Let $A=\left(\mu_{A}, \gamma_{A}\right)$ be an IFS of an LA-ring $R$ with left identity $e$. Then $A$ is an intuitionistic fuzzy ideal of $R$ if and only if $A$ is an intuitionistic fuzzy interior ideal of $R$.

Proof: Let $A=\left(\mu_{A}, \gamma_{A}\right)$ be an intuitionistic fuzzy interior ideal of $R$ and $x, y \in R$. Thus $\mu_{A}(x y)=\mu_{A}((e x) y) \geq \mu_{A}(x)$ and $\gamma_{A}(x y)=\gamma_{A}((e x) y) \leq \gamma_{A}(x)$, i.e., $A$ is an intuitionistic fuzzy right ideal of $R$. Hence $A$ is an intuitionistic fuzzy ideal of $R$ by the Lemma 2.9. Converse is true by the Lemma 2.18 . 
Lemma 2.21: Every intuitionistic fuzzy left (resp. right, two-sided) ideal of an LA-ring $R$ is an intuitionistic fuzzy bi-ideal of $R$.

Proof: Suppose that $A=\left(\mu_{A}, \gamma_{A}\right)$ is an intuitionistic fuzzy right ideal of $R$ and $x, y, z \in R$. Thus

$$
\mu_{A}((x y) z)=\mu_{A}(x y) \geq \mu_{A}(x) \text { and } \mu_{A}((x y) z)=\mu_{A}((z y) x) \geq \mu_{A}(z y) \geq \mu_{A}(z),
$$

this implies that $\mu_{A}((x y) z) \geq \mu_{A}(x) \wedge \mu_{A}(z)$. Similarly, we have $\gamma_{A}((x y) z) \leq \gamma_{A}(x) \vee \gamma_{A}(z)$. Therefore $A$ is an intuitionistic fuzzy bi-ideal of $R$.

The converse is not true in general, giving an example:

Using Example 2.19, $A=\left(\mu_{A}, \gamma_{A}\right)$ is an intuitionistic fuzzy bi-ideal of $R$, but not an intuitionistic fuzzy right ideal of $R$, as

$$
\begin{aligned}
\mu_{A}(41) & =\mu_{A}(3)=0 . \\
\mu_{A}(4) & =0.7 . \\
& \Rightarrow \mu_{A}(41) \geq \mu_{A}(4) . \\
\text { and } \gamma_{A}(41) & =\gamma_{A}(3)=0.7 . \\
\gamma_{A}(4) & =0 . \\
& \Rightarrow \gamma_{A}(41) \pm \gamma_{A}(4) .
\end{aligned}
$$

Lemma 2.22: Every intuitionistic fuzzy bi-ideal of an LA-ring $R$ is an intuitionistic fuzzy generalized bi-ideal of $R$.

Proof: Obvious.

Lemma 2.23: Every intuitionistic fuzzy left ( resp. right, two-sided) ideal of an LA-ring $R$ is an intuitionistic fuzzy quasi-ideal of $R$.

Proof: Assume that $A=\left(\mu_{A}, \gamma_{A}\right)$ is an intuitionistic fuzzy left ideal of $R$. Now $\mu_{A} \circ R \cap R \circ \mu_{A} \subseteq R \circ \mu_{A} \subseteq \mu_{A}$ and $\gamma_{A} \circ R \cup R \circ \gamma_{A} \supseteq R \circ \gamma_{A} \supseteq \gamma_{A}$. So $A$ is an intuitionistic fuzzy quasi-ideal of $R$.

Lemma 2.24: Let $R$ be an LA-ring with left identity $e$, such that $(x e) R=x R$ for all $x \in R$. Then every intuitionistic fuzzy quasi-ideal of $R$ is an intuitionistic fuzzy bi-ideal of $R$.

Proof: Let $A=\left(\mu_{A}, \gamma_{A}\right)$ be an intuitionistic fuzzy quasi-ideal of $R$ and $A \circ A \subseteq A$ by the Proposition 2.20. Now

$$
\begin{aligned}
\left(\mu_{A} \circ R\right) \circ \mu_{A} & \subseteq(R \circ R) \circ \mu_{A} \subseteq R \circ \mu_{A} \\
\text { and }\left(\mu_{A} \circ R\right) \circ \mu_{A} & \subseteq\left(\mu_{A} \circ R\right) \circ R=\left(\mu_{A} \circ R\right) \circ(e \circ R) \\
& =\left(\mu_{A} \circ e\right) \circ(R \circ R) \subseteq\left(\mu_{A} \circ e\right) \circ R=\mu_{A} \circ R . \\
& \Rightarrow\left(\mu_{A} \circ R\right) \circ \mu_{A} \subseteq \mu_{A} \circ R \cap R \circ \mu_{A} \subseteq \mu_{A}
\end{aligned}
$$

Similarly, $\left(\gamma_{A} \circ R\right) \circ \gamma_{A} \supseteq \gamma_{A} \circ R \cup R \circ \gamma_{A} \supseteq \gamma_{A}$. Hence $A$ is an intuitionistic fuzzy bi-ideal of $R$. 


\section{REGULAR LA-RINGS}

An LA-ring $R$ is called a regular if for every $x \in R$, there exists an element $a \in R$ such that $x=(x a) x$. In this section, we characterize regular LA-rings by the properties of intuitionistic fuzzy left ( right, quasi-, bi-, generalized bi-) ideals.

Lemma 3.1: Every intuitionistic fuzzy right ideal of a regular LA-ring $R$ is an intuitionistic fuzzy ideal of $R$.

Proof: Suppose that $A=\left(\mu_{A}, \gamma_{A}\right)$ is an intuitionistic fuzzy right ideal of $R$. Let $x, y \in R$, this implies that there exists an element $a \in R$, such that $x=(x a) x$. Thus

$$
\mu_{A}(x y)=\mu_{A}(((x a) x) y)=\mu_{A}((y x)(x a)) \geq \mu_{A}(y x) \geq \mu_{A}(y)
$$

and

$$
\gamma_{A}(x y)=\gamma_{A}(((x a) x) y)=\gamma_{A}((y x)(x a)) \leq \gamma_{A}(y x) \leq \gamma_{A}(y) .
$$

Hence $A$ is an intuitionistic fuzzy ideal of $R$.

Lemma 3.2: Let $A=\left(\mu_{A}, \gamma_{A}\right)$ be an IFS of a regular LA-ring $R$. Then $A$ is an intuitionistic fuzzy ideal of $R$ if and only if $A$ is an intuitionistic fuzzy interior ideal of $R$.

Proof: Consider that $A=\left(\mu_{A}, \gamma_{A}\right)$ is an intuitionistic fuzzy interior ideal of $R$. Let $x, y \in R$, then there exists an element $a \in R$, such that $x=(x a) x$. Thus

$$
\mu_{A}(x y)=\mu_{A}(((x a) x) y)=\mu_{A}((y x)(x a)) \geq \mu_{A}(x)
$$

and

$$
\gamma_{A}(x y)=\gamma_{A}(((x a) x) y)=\gamma_{A}((y x)(x a)) \leq \gamma_{A}(x),
$$

i.e., $A$ is an intuitionistic fuzzy right ideal of $R$. So $A$ is an intuitionistic fuzzy ideal of $R$ by the Lemma 3.1. Converse is true by the Lemma 2.18.

Remark 3.3: The concept of intuitionistic fuzzy ( interior, two-sided) ideals coincides with the same concept in regular LA-rings.

Proposition 3.4: Let $R$ be a regular LA-ring. Then $(A \circ R) \cap(R \circ A)=A$ for every intuitionistic fuzzy right ideal $A$ of $R$.

Proof: Suppose that $A=\left(\mu_{A}, \gamma_{A}\right)$ is an intuitionistic fuzzy right ideal of $R$. This implies that $(A \circ R) \cap(R \circ A) \subseteq A$, because every intuitionistic fuzzy right ideal of $R$ is an intuitionistic fuzzy quasi-ideal of $R$ by the Lemma 2.23. Let $x \in R$, this implies that there exists an element $a \in R$, such that $x=(x a) x$. Thus

$$
\begin{aligned}
\left(\mu_{A} \circ R\right)(x) & =\vee_{x=\sum_{i=1}^{n} a_{i} b_{i}}\left\{\wedge_{i=1}^{n}\left\{\mu_{A}\left(a_{i}\right) \wedge R\left(b_{i}\right)\right\}\right\} \\
& \geq \mu_{A}(x a) \wedge R(x) \geq \mu_{A}(x) \wedge 1=\mu_{A}(x) \\
\text { and }\left(\gamma_{A} \circ R\right)(x) & =\wedge_{x=\sum_{i=1}^{n} a_{i} b_{i}}\left\{\vee_{i=1}^{n}\left\{\gamma_{A}\left(a_{i}\right) \vee R\left(b_{i}\right)\right\}\right\} \\
& \leq \gamma_{A}(x a) \vee R(x) \leq \gamma_{A}(x) \vee 0=\gamma_{A}(x) . \\
& \Rightarrow A \subseteq A \circ R .
\end{aligned}
$$


Similarly, we have $A \subseteq R \circ A$, i.e., $A \subseteq(A \circ R) \cap(R \circ A)$. Hence $(A \circ R) \cap(R \circ A)=A$.

Lemma 3.5: Let $R$ be a regular LA-ring. Then $D \circ L=D \cap L$ for every intuitionistic fuzzy right ideal $D$ and every intuitionistic fuzzy left ideal $L$ of $R$.

Proof: Since $D \circ L \subseteq D \cap L$, for every intuitionistic fuzzy right ideal $D=\left(\mu_{D}, \gamma_{D}\right)$ and every intuitionistic fuzzy left ideal $L=\left(\mu_{L}, \gamma_{L}\right)$ of $R$ by the Lemma 2.14. Let $x \in R$, this means that there exists an element $a \in R$ such that $x=(x a) x$. Thus

$$
\begin{aligned}
\left(\mu_{D} \circ \mu_{L}\right)(x) & =\vee_{x=\sum_{i=1}^{n} a_{i} b_{i}}\left\{\wedge_{i=1}^{n}\left\{\mu_{D}\left(a_{i}\right) \wedge \mu_{L}\left(b_{i}\right)\right\}\right\} \\
& \geq \mu_{D}(x a) \wedge \mu_{L}(x) \geq \mu_{D}(x) \wedge \mu_{L}(x)=\left(\mu_{D} \cap \mu_{L}\right)(x) \\
\text { and }\left(\gamma_{D} \circ \gamma_{L}\right)(x) & =\wedge_{x=\sum_{i=1}^{n} a_{i} b_{i}}\left\{\vee_{i=1}^{n}\left\{\gamma_{D}\left(a_{i}\right) \vee \gamma_{L}\left(b_{i}\right)\right\}\right\} \\
& \leq \gamma_{D}(x a) \vee \gamma_{L}(x) \leq \gamma_{D}(x) \vee \gamma_{L}(x)=\left(\gamma_{D} \cup \gamma_{L}\right)(x) .
\end{aligned}
$$

Therefore $D \circ L=D \cap L$.

Lemma 3.6: Let $R$ be an LA-ring with left identity $e$. Then $R a$ is the smallest left ideal of $R$ containing $a$.

Proof: Let $x, y \in R a$ and $r \in R$. This implies that $x=r_{1} a$ and $y=r_{2} a$, where $r_{1}, r_{2} \in R$. Now

$$
\begin{aligned}
x-y & =r_{1} a-r_{2} a=\left(r_{1}-r_{2}\right) a \in R a \\
\text { and } r x & =r\left(r_{1} a\right)=(e r)\left(r_{1} a\right)=\left(\left(r_{1} a\right) r\right) e=\left(\left(r_{1} a\right)(e r)\right) e \\
& =\left(\left(r_{1} e\right)(a r)\right) e=(e(a r))\left(r_{1} e\right)=(a r)\left(r_{1} e\right) \\
& =\left(\left(r_{1} e\right) r\right) a \in R a .
\end{aligned}
$$

Since $a=e a \in R a$. Thus $R a$ is a left ideal of $R$ containing $a$. Let $I$ be another left ideal of $R$ containing $a$. Since $r a \in I$, where $r a \in R a$, i.e., $R a \subseteq I$. Hence $R a$ is the smallest left ideal of $R$ containing $a$.

Lemma 3.7: Let $R$ be an LA-ring with left identity $e$. Then $a R$ is a left ideal of $R$.

Proof: Straight forward.

Proposition 3.8: Let $R$ be an LA-ring with left identity $e$. Then $a R \cup R a$ is the smallest right ideal of $R$ containing $a$.

Proof: Let $x, y \in a R \cup R a$, this means that $x, y \in a R$ or $R a$. Since $a R$ and $R a$ both are left ideals of $R$, so $x-y \in a R$ and $R a$, i.e., $x-y \in a R \cup R a$. We have to show that $(a R \cup R a) R \subseteq(a R \cup R a)$. Now

$$
\begin{aligned}
(a R \cup R a) R & =(a R) R \cup(R a) R=(R R) a \cup(R a)(e R) \\
& \subseteq R a \cup(\operatorname{Re})(a R)=R a \cup R(a R) \\
& =R a \cup a(R R) \subseteq R a \cup a R=a R \cup R a . \\
& \Rightarrow(a R \cup R a) R \subseteq a R \cup R a .
\end{aligned}
$$


Since $a \in R a$, i.e., $a \in a R \cup R a$. Let $I$ be another right ideal of $R$ containing $a$. Since $a R \in I R \subseteq I$ and $R a=(R R) a=(a R) R \in(I R) R \subseteq I R \subseteq I$, i.e., $a R \cup R a \subseteq I$. Therefore $a R \cup R a$ is the smallest right ideal of $R$ containing $a$.

Theorem 3.9: Let $R$ be an LA-ring with left identity $e$, such that $(x e) R=x R$ for all $x \in R$. Then the following conditions are equivalent.

(1) $R$ is a regular.

(2) $D \cap L=D \circ L$ for every intuitionistic fuzzy right ideal $D$ and every intuitionistic fuzzy left ideal $L$ of $R$.

(3) $C=(C \circ R) \circ C$ for every intuitionistic fuzzy quasi-ideal $C$ of $R$.

Proof: Suppose that $(1)$ holds and $C=\left(\mu_{C}, \gamma_{C}\right)$ be an intuitionistic fuzzy quasi-ideal of $R$. Then $(C \circ R) \circ C \subseteq C$, because every intuitionistic fuzzy quasi-ideal of $R$ is an intuitionistic fuzzy bi-ideal of $R$ by the Lemma 2.24. Let $x \in R$, this implies that there exists an element $a$ of $R$ such that $x=(x a) x$. Thus

$$
\begin{aligned}
\left(\left(\mu_{C} \circ R\right) \circ \mu_{C}\right)(x) & =\vee_{a=\sum_{i=1}^{n} a_{i} b_{i}}\left\{\wedge_{i=1}^{n}\left\{\left(\mu_{C} \circ R\right)\left(a_{i}\right) \wedge \mu_{C}\left(b_{i}\right)\right\}\right\} \\
& \geq\left(\mu_{C} \circ R\right)(x a) \wedge \mu_{C}(x) \\
& =\vee_{x a=\sum_{i=1}^{n} p_{i} q_{i}}\left\{\wedge_{i=1}^{n}\left\{\mu_{C}\left(p_{i}\right) \wedge R\left(q_{i}\right)\right\}\right\} \wedge \mu_{C}(x) \\
& \geq \mu_{C}(x) \wedge R(a) \wedge \mu_{C}(x)=\mu_{C}(x) . \\
& \Rightarrow \mu_{C} \subseteq\left(\mu_{C} \circ R\right) \circ \mu_{C} .
\end{aligned}
$$

Similarly, we have $\gamma_{C} \supseteq\left(\gamma_{C} \circ R\right) \circ \gamma_{C}$, i.e., $C=(C \circ R) \circ C$. Hence (1) implies (3). Assume that (3) holds. Let $D$ be an intuitionistic fuzzy right ideal and $L$ be an intuitionistic fuzzy left ideal of $R$. This means that $D$ and $L$ be intuitionistic fuzzy quasi-ideals of $R$ by the Lemma 2.23, so $D \cap L$ be also an intuitionistic fuzzy quasi-ideal of $R$. Then by our assumption, $D \cap L=((D \cap L) \circ R) \circ(D \cap L) \subseteq(D \circ R) \circ L \subseteq D \circ L$, i.e., $D \cap L \subseteq D \circ L$. Since $D \circ L \subseteq D \cap L$. Therefore $D \circ L=D \cap L$, i.e., (3) $\Rightarrow(2)$. Suppose that (2) is true and $a \in R$. Then $R a$ is a left ideal of $R$ containing $a$ by the Lemma 3.7 and $a R \cup R a$ is a right ideal of $R$ containing $a$ by the Proposition 3.8. This implies that $\chi_{R a}$ is an intuitionistic fuzzy left ideal and $\chi_{a R \cup R a}$ is an intuitionistic fuzzy right ideal of $R$, by the Theorem 2.3. Then by our supposition

$$
\chi_{a R \cup R a} \cap \chi_{R a}=\chi_{a R \cup R a} \circ \chi_{R a} \text {, i.e., } \quad \chi_{(a R \cup R a) \cap R a}=\chi_{(a R \cup R a) R a}
$$

by the Theorem 2.2. Thus $(a R \cup R a) \cap R a=(a R \cup R a) R a$. Since $a \in(a R \cup R a) \cap R a$, i.e., $a \in(a R \cup R a) R a$, so $a \in(a R)(R a) \cup(R a)(R a)$. This implies that

$$
a \in(a R)(R a) \text { or } a \in(R a)(R a)
$$

If $a \in(a R)(R a)$, then 
$a=(a x)(y a)=((y a) x) a=(((e y) a) x) a=(((a y) e) x) a=((x e)(a y)) a=(a((x e) y)) a$ for any $x, y \in R$.

If $a \in(R a)(R a)$, then $\quad(R a)(R a)=((\operatorname{Re}) a)(R a)=((a e) R)(R a)=(a R)(R a), \quad$ i.e., $a \in(a R)(R a)$. So $a$ is a regular, i.e., $R$ is a regular. Hence $(2) \Rightarrow(1)$.

Theorem 3.10: Let $R$ be an LA-ring with left identity $e$, such that $(x e) R=x R$ for all $x \in R$. Then the following conditions are equivalent.

(1) $R$ is a regular.

(2) $A=(A \circ R) \circ A$ for every intuitionistic fuzzy quasi-ideal $A$ of $R$.

(3) $B=(B \circ R) \circ B$ for every intuitionistic fuzzy bi-ideal $B$ of $R$.

(4) $C=(C \circ R) \circ C$ for every intuitionistic fuzzy generalized bi-ideal $C$ of $R$.

Proof: $(1) \Rightarrow(4)$, is obvious. Since $(4) \Rightarrow(3)$, every intuitionistic fuzzy bi-ideal of $R$ is an intuitionistic fuzzy generalized bi-ideal of $R$ by the Lemma 2.22. Since (3) $\Rightarrow(2)$, every intuitionistic fuzzy quasi-ideal of $R$ is an intuitionistic fuzzy bi-ideal of $R$ by the Lemma 15. $(2) \Rightarrow(1)$, by the Theorem 3.9 .

Theorem 3.11: Let $R$ be an LA-ring with left identity $e$, such that $(x e) R=x R$ for all $x \in R$. Then the following conditions are equivalent.

(1) $R$ is a regular.

(2) $A \cap I=(A \circ I) \circ A$ for every intuitionistic fuzzy quasi-ideal $A$ and every intuitionistic fuzzy ideal $I$ of $R$.

(3) $B \cap I=(B \circ I) \circ B$ for every intuitionistic fuzzy bi-ideal $B$ and every intuitionistic fuzzy ideal $I$ of $R$.

(4) $C \cap I=(C \circ I) \circ C$ for every intuitionistic fuzzy generalized bi-idea $C$ and every intuitionistic fuzzy ideal $I$ of $R$.

Proof: Assume that $(1)$ holds. Let $C=\left(\mu_{C}, \gamma_{C}\right)$ be an intuitionistic fuzzy generalized biideal and $I=\left(\mu_{I}, \gamma_{I}\right)$ be an intuitionistic fuzzy ideal of $R$. Now $(C \circ I) \circ C \subseteq(R \circ I) \circ R \subseteq I \circ R \subseteq I \quad$ and $\quad(C \circ I) \circ C \subseteq(C \circ R) \circ C \subseteq C, \quad$ i.e. $(C \circ I) \circ C \subseteq C \cap I$. Let $x \in R$, this means that there exists an element $a \in R$ such that $x=(x a) x$. Now $x a=((x a) x) a=(a x)(x a)=x((a x) a)$. Thus

$$
\begin{aligned}
\left(\left(\mu_{C} \circ \mu_{I}\right) \circ \mu_{C}\right)(x) & =\vee_{x=\sum_{i=1}^{n} a_{i} b_{i}}\left\{\wedge_{i=1}^{n}\left\{\left(\mu_{C} \circ \mu_{I}\right)\left(a_{i}\right) \wedge \mu_{C}\left(b_{i}\right)\right\}\right\} \\
& \geq\left(\mu_{C} \circ \mu_{I}\right)(x a) \wedge \mu_{C}(x) \\
& =\vee_{x a=\sum_{i=1}^{n} p_{i} q_{i}}\left\{\wedge_{i=1}^{n}\left\{\mu_{C}\left(p_{i}\right) \wedge \mu_{I}\left(q_{i}\right)\right\}\right\} \wedge \mu_{C}(x) \\
& \geq \mu_{C}(x) \wedge \mu_{I}((a x) a) \wedge \mu_{C}(x) \\
& \geq \mu_{C}(x) \wedge \mu_{I}(x)=\left(\mu_{C} \cap \mu_{I}\right)(x) . \\
& \Rightarrow \mu_{C} \cap \mu_{I} \subseteq\left(\mu_{C} \circ \mu_{I}\right) \circ \mu_{C} .
\end{aligned}
$$

Similarly, we have $\gamma_{C} \cup \gamma_{I} \supseteq\left(\gamma_{C} \circ \gamma_{I}\right) \circ \gamma_{C}$. Hence $C \cap I=(C \circ I) \circ C$, i.e., $(1) \Rightarrow(4)$. It is clear that $(4) \Rightarrow(3)$ and $(3) \Rightarrow(2)$. Suppose that (2) is true. Then 
$A \cap R=(A \circ R) \circ A$, where $R$ itself is an intuitionistic fuzzy two-sided ideal of $R$. So $A=(A \circ R) \circ A$, because every intuitionistic fuzzy two-sided ideal of $R$ is an intuitionisitc fuzzy quasi-ideal of $R$. Hence $R$ is a regular by the Theorem 3.9, i.e., $(2) \Rightarrow(1)$.

Theorem 3.12: Let $R$ be an LA-ring with left identity $e$, such that $(x e) R=x R$ for all $x \in R$. Then the following conditions are equivalent.

(1) $R$ is a regular.

(2) $A \cap D \subseteq D \circ A$ for every intuitionistic fuzzy quasi-ideal $A$ and every intuitionistic fuzzy right ideal $D$ of $R$.

(3) $B \cap D \subseteq D \circ B$ for every intuitionistic fuzzy bi-ideal $B$ and every intuitionistic fuzzy right ideal $D$ of $R$.

(4) $C \cap D \subseteq D \circ C$ for every intuitionistic fuzzy generalized bi-ideal $C$ and every intuitionistic fuzzy right ideal $D$ of $R$.

Proof: $(1) \Rightarrow(4)$, is obvious. It is clear that (4) $\Rightarrow$ (3) and (3) $\Rightarrow(2)$. Suppose that (2) holds, this implies that $D \cap A=A \cap D \subseteq D \circ A$, where $A$ is an intuitionistic fuzzy left ideal of $R$. Since $D \circ A \subseteq D \cap A$, i.e., $D \cap A=D \circ A$. Hence $R$ is a regular by the Theorem 3.9, i.e., $(2) \Rightarrow(1)$.

\section{IINTRA-REGULAR LA-RINGS}

An LA-ring An LA-ring $R$ is called an intra-regular if for every $x \in R$, there exist elements $a_{i}, b_{i} \in R$ such that $x=\sum_{i=1}^{n}\left(a_{i} x^{2}\right) b_{i}$. In this section, we characterize intraregular LA-rings by the properties of intuitionistic fuzzy left ( right, quasi-, bi-, generalized bi- ) ideals.

Lemma 4.1: Every intuitionistic fuzzy left ( right) ideal of an intra-regular LA-ring $R$ is an intuitionistic fuzzy ideal of $R$.

Proof: Suppose that $A=\left(\mu_{A}, \gamma_{A}\right)$ is an intuitionistic fuzzy right ideal of $R$. Let $x, y \in R$, this implies that there exist elements $a_{i}, b_{i} \in R$, such that $x=\sum_{i=1}^{n}\left(a_{i} x^{2}\right) b_{i}$. Thus

$$
\begin{aligned}
\mu_{A}(x y) & =\mu_{A}\left(\left(\left(a_{i} x^{2}\right) b_{i}\right) y\right)=\mu_{A}\left(\left(y b_{i}\right)\left(a_{i} x^{2}\right)\right) \\
& \geq \mu_{A}\left(y b_{i}\right) \geq \mu_{A}(y) \\
\text { and } \gamma_{A}(x y) & =\gamma_{A}\left(\left(\left(a_{i} x^{2}\right) b_{i}\right) y\right)=\gamma_{A}\left(\left(y b_{i}\right)\left(a_{i} x^{2}\right)\right) \\
& \leq \gamma_{A}\left(y b_{i}\right) \leq \gamma_{A}(y) .
\end{aligned}
$$

Hence $A$ is an intuitionistic fuzzy ideal of $R$.

Proposition 4.2: Let $A$ be an IFS of an intra-regular LA-ring $R$ with left identity $e$. Then $A$ is an intuitionistic fuzzy ideal of $R$ if and only if $A$ is an intuitionistic fuzzy interior ideal of $R$.

Proof: Suppose that $A=\left(\mu_{A}, \gamma_{A}\right)$ is an intuitionistic fuzzy interior ideal of $R$. Let $x, y \in R$, this implies that there exist elements $a_{i}, b_{i} \in R$, such that $x=\sum_{i=1}^{n}\left(a_{i} x^{2}\right) b_{i}$. Thus 


$$
\begin{aligned}
\mu_{A}(x y) & =\mu_{A}\left(\left(\left(a_{i} x^{2}\right) b_{i}\right) y\right)=\mu_{A}\left(\left(y b_{i}\right)\left(a_{i} x^{2}\right)\right) \\
& =\mu_{A}\left(\left(y b_{i}\right)\left(a_{i}(x x)\right)\right)=\mu_{A}\left(\left(y b_{i}\right)\left(x\left(a_{i} x\right)\right)\right) \\
& =\mu_{A}\left((y x)\left(b_{i}\left(a_{i} x\right)\right)\right) \geq \mu_{A}(x) .
\end{aligned}
$$

Similarly, we have $\gamma_{A}(x y) \leq \gamma_{A}(x)$, i.e., $A$ is an intuitionistic fuzzy right ideal of $R$. Hence $A$ is an intuitionistic fuzzy ideal of $R$ by the Lemma 4.1. Converse is true by the Lemma 2.18 .

Remark 4.3: The concept of intuitionistic fuzzy ( interior, two-sided) ideals coincides in intra-regular LA-rings with left identity.

Lemma 4.4: Let $R$ be an intra-regular LA-ring with left identity $e$. Then $D \cap L \subseteq L \circ D$ for every intuitionistic fuzzy left ideal $L$ and every intuitionistic fuzzy right ideal $D$ of $R$.

Proof: Let $L=\left(\mu_{L}, \gamma_{L}\right)$ be an intuitionistic fuzzy left ideal and $D=\left(\mu_{D}, \gamma_{D}\right)$ be an intuitionistic fuzzy right ideal of $R$. Let $x \in R$, this means that there exist elements such that $a_{i}, b_{i} \in R$ such that $x=\sum_{i=1}^{n}\left(a_{i} x^{2}\right) b_{i}$. Now

$$
\begin{aligned}
x & =\left(a_{i} x^{2}\right) b_{i}=\left(a_{i}(x x)\right) b_{i}=\left(x\left(a_{i} x\right)\right) b_{i} \\
& =\left(x\left(a_{i} x\right)\right)\left(e b_{i}\right)=(x e)\left(\left(a_{i} x\right) b_{i}\right)=\left(a_{i} x\right)\left((x e) b_{i}\right) .
\end{aligned}
$$

Thus

$$
\begin{aligned}
\left(\mu_{L} \circ \mu_{D}\right)(x) & =\vee_{x=\sum_{i=1}^{n} p_{i} q_{i}}\left\{\wedge_{i=1}^{n}\left\{\mu_{L}\left(p_{i}\right) \wedge \mu_{D}\left(q_{i}\right)\right\}\right\} \\
& \geq \mu_{L}\left(a_{i} x\right) \wedge \mu_{D}\left((x e) b_{i}\right) \geq \mu_{L}(x) \wedge \mu_{D}(x) \\
& =\mu_{D}(x) \wedge \mu_{L}(x)=\left(\mu_{D} \cap \mu_{L}\right)(x) \\
\text { and }\left(\gamma_{L} \circ \gamma_{D}\right)(x) & =\wedge_{x=\sum_{i=1}^{n} p_{i} q_{i}}\left\{\vee_{i=1}^{n}\left\{\gamma_{L}\left(p_{i}\right) \vee \gamma_{D}\left(q_{i}\right)\right\}\right\} \\
& \leq \gamma_{L}\left(a_{i} x\right) \vee \gamma_{D}\left((x e) b_{i}\right) \leq \gamma_{L}(x) \vee \gamma_{D}(x) \\
& =\gamma_{D}(x) \vee \gamma_{L}(x)=\left(\gamma_{D} \cup \gamma_{L}\right)(x) . \\
& \Rightarrow D \cap L \subseteq L \circ D .
\end{aligned}
$$

Theorem 4.5: Let $R$ be an LA-ring with left identity $e$, such that $(x e) R=x R$ for all $x \in R$. Then the following conditions are equivalent.

(1) $R$ is an intra-regular.

(2) $D \cap L \subseteq L \circ D$ for every intuitionistic fuzzy left ideal $L$ and every intuitionistic fuzzy right ideal $D$ of $R$.

Proof: $(1) \Rightarrow(2)$ is true by the Lemma 4.4. Suppose that (2) holds. Let $a \in R$, then $R a$ is a left ideal of $R$ containing $a$ by the Lemma 3.6 and $a R \cup R a$ is a right ideal of $R$ containing $a$ by the Proposition 3.8. So $\chi_{R a}$ is an intuitionistic fuzzy left ideal and $\chi_{a R \cup R a}$ is an intuitionistic fuzzy right ideal of $R$, by the Theorem 1.3. By our supposition

$$
\chi_{a R \cup R a} \cap \chi_{R a} \subseteq \chi_{R a} \circ \chi_{a R \cup R a} \text {, i.e., } \chi_{(a R \cup R a) \cap R a} \subseteq \chi_{(R a)(a R \cup R a)}
$$


by the Theorem 1.2. Thus $(a R \cup R a) \cap R a \subseteq R a(a R \cup R a)$. Since $a \in(a R \cup R a) \cap R a$, i.e., $a \in R a(a R \cup R a)=(R a)(a R) \cup(R a)(R a)$. This implies that $a \in(R a)(a R)$ or $a \in(R a)(R a)$. If $a \in(R a)(a R)$, then

$$
\begin{aligned}
(R a)(a R) & =(R a)((e a)(R R))=(R a)((R R)(a e)) \\
& =(R a)(((a e) R) R)=(R a)((a R) R) \\
& =(R a)((R R) a)=(R a)(R a)=((R a) a) R \\
& =((R a)(e a)) R=((\operatorname{Re})(a a)) R=\left(R a^{2}\right) R .
\end{aligned}
$$

So $a \in\left(R a^{2}\right) R$. If $a \in(R a)(R a)$, then obvious $a \in\left(R a^{2}\right) R$. This implies that $a$ is an intraregular. Hence $R$ is an intra-regular, i.e., $(2) \Rightarrow(1)$.

Theorem 4.6: Let $R$ be an LA-ring with left identity $e$, such that $(x e) R=x R$ for all $x \in R$. Then the following conditions are equivalent.

(1) $R$ is an intra-regular.

(2) $A \cap I=(A \circ I) \circ A$ for every intuitionistic fuzzy quasi-ideal $A$ and every intuitionistic fuzzy ideal $I$ of $R$.

(3) $B \cap I=(B \circ I) \circ B$ for every intuitionistic fuzzy bi-ideal $B$ and every intuitionistic fuzzy ideal $I$ of $R$.

(4) $C \cap I=(C \circ I) \circ C$ for every intuitionistic fuzzy generalized bi-ideal $C$ and every intuitionistic fuzzy ideal $I$ of $R$.

Proof: Suppose that $(1)$ holds. Let $C=\left(\mu_{C}, \gamma_{C}\right)$ be an intuitionistic fuzzy generalized bi-ideal and $I=\left(\mu_{I}, \gamma_{I}\right)$ be an intuitionistic fuzzy ideal of $R$. Now $(C \circ I) \circ C \subseteq(R \circ I) \circ R \subseteq I \circ R \subseteq I \quad$ and $\quad(C \circ I) \circ C \subseteq(C \circ R) \circ C \subseteq C, \quad$ thus $(C \circ I) \circ C \subseteq C \cap I$. Let $x \in R$, this implies that there exist elements $a_{i}, b_{i} \in R$ such that $x=\sum_{i=1}^{n}\left(a_{i} x^{2}\right) b_{i}$. Now

$$
\begin{aligned}
x & =\left(a_{i} x^{2}\right) b_{i}=\left(a_{i}(x x)\right) b_{i}=\left(x\left(a_{i} x\right)\right) b=\left(b_{i}\left(a_{i} x\right)\right) x . \\
b_{i}\left(a_{i} x\right) & =b_{i}\left(a_{i}\left(\left(a_{i} x^{2}\right) b_{i}\right)\right)=b_{i}\left(\left(a_{i} x^{2}\right)\left(a_{i} b_{i}\right)\right)=b_{i}\left(\left(a_{i} x^{2}\right) c_{i}\right) \\
& =\left(a_{i} x^{2}\right)\left(b_{i} c_{i}\right)=\left(a_{i} x^{2}\right) d_{i}=\left(a_{i} x^{2}\right)\left(e d_{i}\right)=\left(d_{i} e\right)\left(x^{2} a_{i}\right) \\
& =m_{i}\left(x^{2} a_{i}\right)=x^{2}\left(m_{i} a_{i}\right)=(x x) l_{i}=\left(l_{i} x\right) x=\left(l_{i} x\right)(e x) \\
& =(x e)\left(x l_{i}\right)=x\left((x e) l_{i}\right) .
\end{aligned}
$$

Thus

$$
\begin{aligned}
\left(\left(\mu_{C} \circ \mu_{I}\right) \circ \mu_{C}\right)(x) & =\vee_{x=\sum_{i=1}^{n} p_{i} q_{i}}\left\{\wedge_{i=1}^{n}\left\{\left(\mu_{C} \circ \mu_{I}\right)\left(p_{i}\right) \wedge \mu_{C}\left(q_{i}\right)\right\}\right\} \\
& \geq\left(\mu_{C} \circ \mu_{I}\right)\left(b_{i}\left(a_{i} x\right)\right) \wedge \mu_{C}(x) \\
& =\vee_{b_{i}\left(a_{i} x\right)=\sum_{i=1}^{n} m_{i} n_{i}}\left\{\wedge_{i=1}^{n}\left\{\mu_{C}\left(m_{i}\right) \wedge \mu_{I}\left(n_{i}\right)\right\}\right\} \wedge \mu_{C}(x) \\
& \geq \mu_{C}(x) \wedge \mu_{I}\left((x e) l_{i}\right) \wedge \mu_{C}(x) \\
& \geq \mu_{C}(x) \wedge \mu_{I}(x)=\left(\mu_{C} \cap \mu_{I}\right)(x) . \\
& \Rightarrow \mu_{C} \cap \mu_{I} \subseteq\left(\mu_{C} \circ \mu_{I}\right) \circ \mu_{C} .
\end{aligned}
$$


Similarly, we have $\gamma_{C} \cup \gamma_{I} \supseteq\left(\gamma_{C} \circ \gamma_{I}\right) \circ \gamma_{C}$. Hence $C \cap I=(C \circ I) \circ C$, i.e. (1) $\Rightarrow(4)$. It is clear that $(4) \Rightarrow(3)$ and (3) $\Rightarrow(2)$. Assume that (2) is true. Let $A$ be an intuitionistic fuzzy right ideal and $I$ be an intuitionistic fuzzy two-sided ideal of $R$. Since every intuitionistic fuzzy right ideal of $R$ is an intuitionistic fuzzy quasi-ideal of $R$ by the Lemma 2.23, so $A$ is an intuitionistic fuzzy quasi-ideal of $R$. By our assumption $A \cap I=(A \circ I) \circ A \subseteq(R \circ I) \circ A \subseteq I \circ A$, i.e., $A \cap I \subseteq I \circ A$. Hence $R$ is an intra-regular by the Theorem 4.5 , i.e., $(2) \Rightarrow(1)$.

Theorem 4.7: Let $R$ be an LA-ring with left identity $e$, such that $(x e) R=x R$ for all $x \in R$. Then the following conditions are equivalent.

(1) $R$ is an intra-regular.

(2) $A \cap L \subseteq L \circ A$ for every intuitionistic fuzzy quasi-ideal $A$ and every intuitionistic fuzzy left ideal $L$ of $R$.

(3) $B \cap L \subseteq L \circ B$ for every intuitionistic fuzzy bi-ideal $B$ and every intuitionistic fuzzy left ideal $L$ of $R$.

(4) $C \cap L \subseteq L \circ C$ for every intuitionistic fuzzy generalized bi-ideal $C$ and every intuitionistic fuzzy left ideal $L$ of $R$.

Proof: Assume that $(1)$ holds. Let $C=\left(\mu_{C}, \gamma_{C}\right)$ be an intuitionistic fuzzy generalized bi-ideal and $L=\left(\mu_{L}, \gamma_{L}\right)$ be an intuitionistic fuzzy left ideal of $R$. Let $x \in R$, this means that there exist elements $a_{i}, b_{i} \in R \quad$ such that $x=\sum_{i=1}^{n}\left(a_{i} x^{2}\right) b_{i}$. Now $x=\left(a_{i}(x x)\right) b_{i}=\left(x\left(a_{i} x\right)\right) b_{i}=\left(b_{i}\left(a_{i} x\right)\right) x$. Thus

$$
\begin{aligned}
\left(\mu_{L} \circ \mu_{C}\right)(x) & =\vee_{x=\sum_{i=1}^{n} p_{i} q_{i}}\left\{\wedge_{i=1}^{n}\left\{\mu_{L}\left(p_{i}\right) \wedge \mu_{C}\left(q_{i}\right)\right\}\right\} \\
& \geq \mu_{L}\left(b_{i}\left(a_{i} x\right)\right) \wedge \mu_{C}(x) \geq \mu_{L}(x) \wedge \mu_{C}(x) \\
& =\mu_{C}(x) \wedge \mu_{L}(x)=\left(\mu_{C} \cap \mu_{L}\right)(x) . \\
& \Rightarrow \mu_{C} \cap \mu_{L} \subseteq \mu_{L} \circ \mu_{C} .
\end{aligned}
$$

Similarly, we have $\gamma_{C} \cup \gamma_{L} \supseteq \gamma_{L} \circ \gamma_{C}$. Hence $C \cap L \subseteq L \circ C$, i.e., (1) $\Rightarrow$ (4). It is clear that $(4) \Rightarrow(3)$ and $(3) \Rightarrow(2)$. Suppose that (2) holds. Let $A$ be an intuitionistic fuzzy right ideal and $L$ be an intuitionistic fuzzy left ideal of $R$. Since every intuitionistic fuzzy right ideal of $R$ is an intuitionistic fuzzy quasi-ideal of $R$, this implies that $A$ is an intuitionistic fuzzy quasi-ideal of $R$. By our supposition , $A \cap L \subseteq L \circ A$. Thus $R$ is an intra-regular by the Theorem 4.5 , i.e., $(2) \Rightarrow(1)$.

\section{CONCLUSION}

Our ambition is to inspire the study and maturity of non associative algebraic structure (LA-ring). The objective is to explain original methodological developments on ordered LArings, which will be very helpful for upcoming theory of algebraic structure. The ideal of fuzzy set to the characterizations of LA-rings are captivating a great attention of algebraist. 
The aim of this paper is to investigate, the study of (regular, intra-regular) LA-rings by using of fuzzy left (right, interior, quasi-, bi-, generalized bi-) ideals.

\section{Acknowledgements:}

The authors thank the anonymous referees for their careful reading and insightful suggestions.

\section{REFERENCES}

[1] K. T. Atanassov, "New operations defined over the intuitionistic fuzzy sets", Fuzzy Sets and Systems, 61, 137-142 (1994).

[2] B. Banerjee, D. K. Basnet, "Intuitionistic fuzzy subrings and ideals", J. Fuzzy Math., 11, 139-155 (2003).

[3] K. A. Dib, N. Galhum, A. A. M. Hassan, "Fuzzy rings and fuzzy ideals", Fuzzy Math., 4, 245-261 (1996).

[4] K. C. Gupta, M. K. Kantroo, "The intrinsic product of fuzzy subsets of a ring", Fuzzy Sets and Systems, 57, 103-110 (1993).

[5] K. Hur, S. Y. Jang, H. W. Kang, "Intuitionistic fuzzy ideals of a ring", J.Korea Soc. Math. Educ. Ser. B: Pure Appl. Math., 12, 193-209, (2005).

[6] K. Hur, H. W. Kang, H. k. Song, "Intuitionistic fuzzy subgroups and subrings", Honam Math. J., 25, 19-41 (2003).

[7] M. A. Kazim, M. Naseerudin, "On almost semigroups", Alig. Bull. Math., 2, 1-7, (1972).

[8] N. Kausar, M. Waqar, "Characterizations of non-associative rings by their intuitionistic fuzzy biideals", European Journal of Pure and Applied Mathematics, 1, 226-250 (2019).

[9] N. Kausar, "Characterizations of non-associative ordered semi-groups by the properties of their fuzzy ideals with thresholds ( $\alpha, \beta]$ ]", Prikladnaya Diskretnaya Matematika, 4, 337-59 (2019).

[10] N. Kausar, "Direct product of finite intuitionistic fuzzy normal subrings over non-associative rings", European Journal of Pure and Applied Mathematics, 12(2), 622-648 (2019).

[11] N. Kausar, B. Islam, M. Javaid, S, Amjad, U. Ijaz, "Characterizations of non-associative rings by the properties of their fuzzy ideals", Journal of Taibah University for Science, 13 (1), 820-833 (2019).

[12] N. Kausar, B. Islam, S. Amjad, M. Waqar, "Intuitionistics fuzzy ideals with thresholds $(\alpha, \beta]$ in LA-rings", European Journal of Pure and Applied Mathematics, 12 (3), 906-943 (2019).

[13] N. Kausar, M. Waqar, "Direct product of finite fuzzy normal subrings over non-associative rings"” International Journal of Analysis and Applications, 17(5), 752-770 (2019).

[14] N. Kausar, M. Alesemi, Salahuddin, "Anti-fuzzy interior ideals on Ordered AG-groupoids", European Journal of Pure and Applied Mathematics, 13 (1), 113-129 (2020).

[15] N. Kausar, Meshari Alesemi, Salahuddin, Mohammad Munir, "Characterizations of nonassociative ordered semigroups by their intuitionistic fuzzy bi-ideals", Discontinuity, Nonlinearity, and Complexity, 9 (2), 257-275 (2020).

[16] M. S. Kamran, Conditions for LA-semigroups to resemble associative structures, Ph.D. Thesis, Quaid-i-Azam University, Islamabad, (1993).

[17] N. Kuroki, "Regular fuzzy duo rings", Inform. Sci., 94, 119-139 (1996).

[18] W. J. Liu, "Fuzzy invariant subgroups and ideals", Fuzzy Sets and Systems, 8, 133-139 (1982).

[19] T. K. Mukherjee, M. K. Sen, "On fuzzy ideals of a ring 1", Fuzzy Sets and Systems, 21, 99-104 (1987).

[20] T. K. Mukherjee, M. K. Sen, "Prime fuzzy ideals in rings", Fuzzy Sets and Systems, 32, 337-341 (1989).

[21] T. Shah, N. Kausar, "Characterizations of non-associative ordered semigroups by their fuzzy biideals", Theoretical Computer Science, 529, 96-110 (2014). 
[22] T. Shah, N. Kausar, I. Rehman, "Intuitionistic fuzzy normal subrings over a non-associative ring”, An. St. Univ. Ovidius Constanta, 1, 369-386 (2012).

[23] U. M. Swamy, K. L. N. Swamy, "Fuzzy prime ideals of rings", J. Math. Anal. Appl., 134, 94-103 (1988).

[24] L. A. Zadeh, "Fuzzy sets”, Information and control, 8, 338-363 (1965).

Received July 27, 2019 